\title{
Adenocarcinoma y tumor del estroma gastrointestinal del estómago (GIST) sincrónicos
}

\author{
Synchronous adenocarcinoma and gastrointestinal stromal tumor \\ of the stomach (GIST)
}

Josefina Sáez B. ${ }^{1}$, Natalia Gómez A. ${ }^{2}$, Javiera Torres M. ${ }^{3}$ y Mauricio Gabrielli N. ${ }^{4}$

Mujer de 73 años, hipertensa, estudiada por anemia asintomática. Destacaba una masa palpable en hipocondrio izquierdo.

La endoscopía mostró una lesión antral Bormann III y la biopsia un adenocarcinoma.

La etapificación reveló una masa exofítica, heterogénea y que captaba contraste, adyacente a la curvatura menor gástrica, sugerente de un tumor del estroma gastrointestinal (GIST) (Figura 1).

Se realizó una gastrectomía subtotal abierta.

La macroscopía describe un tumor gástrico y un nódulo sólido que protruía por la superficie externa (Figura 2). La biopsia confirmó un adenocarcinoma tubular moderadamente diferenciado que invadía peritoneo visceral, sin permeaciones ni compromiso ganglionar (Figura 3). El nódulo era un GIST con índice mitótico bajo (Figura 4) e inmunohistoquímica compatible.

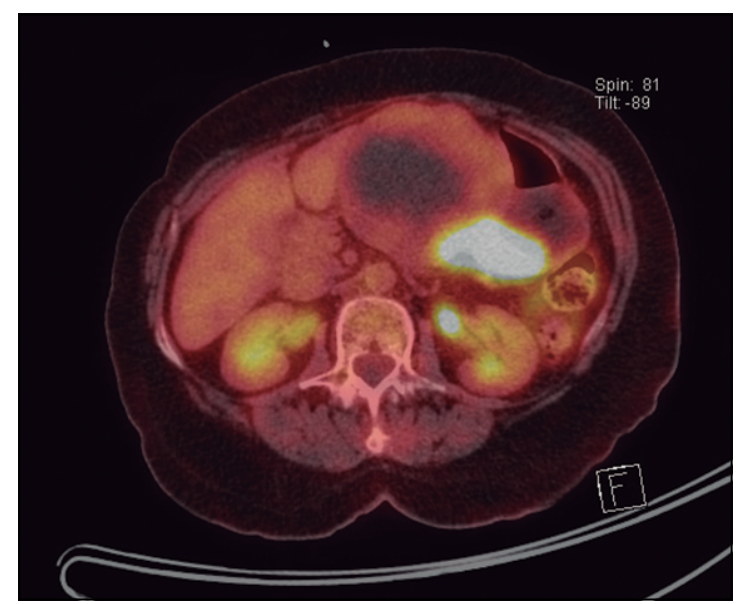

Figura 1. F18-FDG PET CT. Se observa el engrosamiento parietal hipermetabólico compatible con una neoplasia gástrica primaria y una masa exofítica con discreto aumento del metabolismo glucídico.
$50-60 \%$ de los GIST son gástricos, representando $1 \%$ de las neoplasias malignas de este órgano. Suelen ser incidentales y son raramente sincrónicos con otras neoplasias gástricas ${ }^{1-4}$.

$20 \%$ de los pacientes con GIST desarrollan otros cánceres ${ }^{4}$; mutaciones genéticas explicarían la predisposición al desarrollo de neoplasias sincrónicas ${ }^{3-4}$.

La histopatología es fundamental para el diagnóstico, estratificar riesgo y predecir la recurrencia ${ }^{4}$. El pilar del tratamiento es quirúrgico ${ }^{5}$, pero al seleccionar adyuvancia, la etapificación y agresividad de algunos tumores tienen mayor importancia en el pronóstico y deben comandar las decisiones terapéuticas en neoplasias sincrónicas.

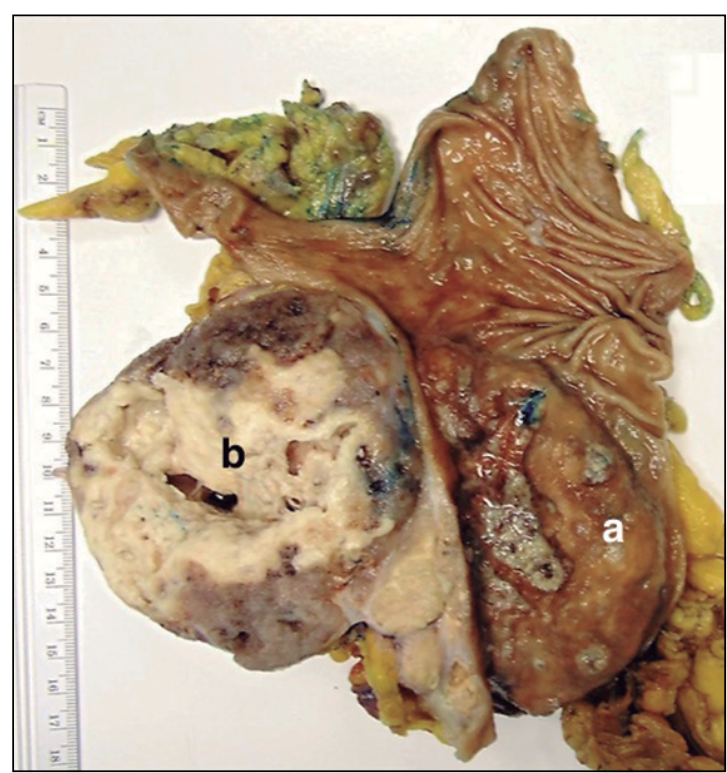

Figura 2. Macroscopía. Tumor exofítico, anular y ulcerado en el antro gástrico (a) y lesión adyacente con bordes expansivos y necrosis central (b).
'Residente Cirugía General, Facultad de Medicina, Pontificia Universidad Católica de Chile.

2Residente Anatomía Patológica, Facultad de Medicina, Pontificia Universidad Católica de Chile. ${ }^{3}$ Departamento de Anatomía Patológica, Facultad de Medicina, Pontificia Universidad Católica de Chile. ${ }^{4}$ Departamento de Cirugía Digestiva, División de Cirugía, Facultad de Medicina,

Pontificia Universidad Católica de Chile.

Recibido el 8 de septiembre de 2017, aceptado para publicación el 23 de noviembre de 2017.

Correspondencia a: Mauricio Gabrielli N. Departamento de Cirugía Digestiva, División de Cirugía, Facultad de Medicina, Pontificia Universidad Católica de Chile

Diagonal Paraguay 362, $4^{\circ}$ piso, Santiago 8330033 , Chile.

maurogabrielli@gmail.com 


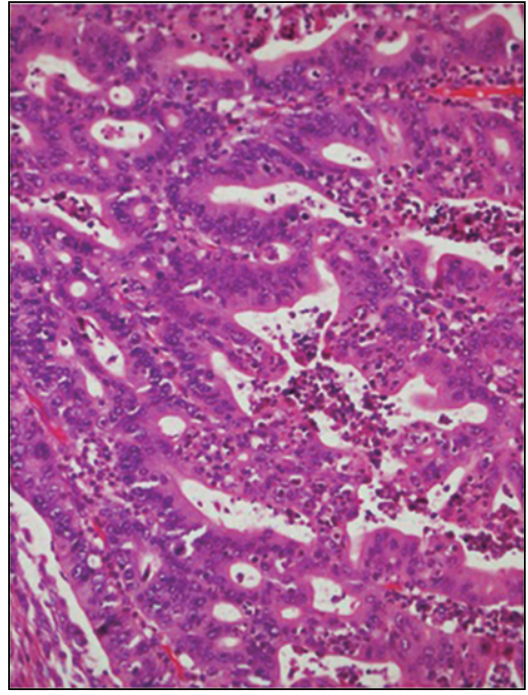

Figura 3. Microscopía. Hematoxilina-eosina. Adenocarcinoma moderadamente diferenciado.

\section{Responsabilidades éticas}

Protección de personas y animales. Los autores declaran que para esta investigación no se han realizado experimentos en seres humanos ni en animales.

Confidencialidad de los datos. Los autores declaran que han seguido los protocolos de su centro de trabajo sobre la publicación de datos de pacientes.

Derecho a la privacidad y consentimiento in-

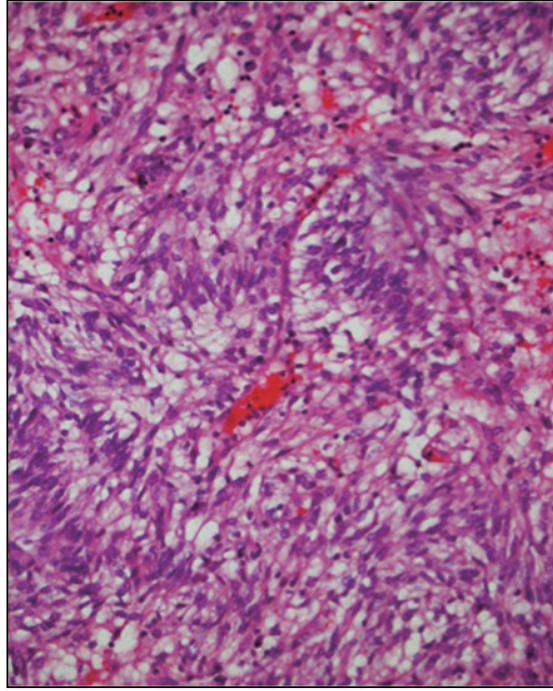

Figura 4. Microscopía. Hematoxilina-eosina. Neoplasia fusocelular de moderada celularidad, sin atipias ni actividad mitótica.

formado. Los autores declaran que en este artículo no aparecen datos de pacientes.

\section{Conflicto de intereses}

Los autores no tienen conflicto de intereses que declarar.

\section{Financiación}

El trabajo no tuvo fuente de financiamiento.

\section{Bibliografía}

1. Sista F, Abruzzese V, Schietroma M, Amicucci G. Concomitant Gastrointestinal Stromal Tumor of the Stomach and Gastric Adenocarcinoma in a Patient with Billroth 2 Resection. Case Reports in Surgery 2013;2013:583856.

2. Maiorana A, Fante R, Cesinaro M, Fano R. Synchronous occurrence of epithelial and stromal tumors in the stomach: a report of 6 cases. Arch Pathol Lab Med. 2000;124: 682-6.

3. Yamamoto D, Hamada Y, Tsubota Y, Kwakami K, Yamamoto Ch, Yamamoto M. Simultaneous development of adenocarcinoma and gastrointestinal stromal tumor (GIST) in the stomach: case report. WJ Surg Oncol. 2012;10.

4. Telugu R, Pshparaj M, Masih D,
Pulimood A. Synchronous appearance of adenocarcinoma and gastrointestinal stromal tumour (GIST) of the stomach: a case report. J Clin and Diagn Res. 2016;10(2) ED16-ED18.

5. Cai R, Ren G, Wang D-B. Synchronous adenocarcinoma and gastrointestinal stromal tumors in the stomach. World J Gastroenterol. 2013;19: 3117-23. 